COMMUNICATIONS IN

ANALYSIS AND GEOMETRY

Volume 13, Number 5, 1039-1055, 2005

\title{
Diameter control under Ricci flow
}

\author{
Peter Topping ${ }^{1}$
}

We estimate the diameter of a closed manifold evolving under Ricci flow in terms of a scalar curvature integral. The proof uses a new maximal function and extends some of Perelman's recent ideas.

\section{Introduction.}

Given a closed manifold $\mathcal{M}$, a smooth family of Riemannian metrics $g(t)$ for $t \in[0, T]$ is said to be evolving under Ricci flow if

$$
\frac{\partial g}{\partial t}=-2 \operatorname{Ric}(g)
$$

This geometric flow was introduced by Hamilton [2] together with the 'normalised Ricci flow'

$$
\frac{\partial g}{\partial t}=-2 \operatorname{Ric}(g)+\frac{2}{n}(f R d V) g
$$

in which the volume is kept fixed by allowing the metric to drift by an appropriate homothetic scaling. Here, Ric and $R$ are the Ricci and scalar curvature respectively. The two flows then differ only by a space-scaling together with a reparameterisation of time. Given an 'initial' metric $g_{0}$, Hamilton [2] and DeTurck [1] proved that there is a unique solution to these flows over some short time interval, with $g(0)=g_{0}$.

As the metric evolves during these flows, the distance between two points, and in particular, the diameter of the manifold, is liable to change. If one takes $M$ to be an upper bound for $\mid$ Ric $\mid$ throughout $\mathcal{M} \times[0, T]$, then one obtains some control immediately from the equation (1.1): for any vector $X$, we have

$$
\left|\frac{\partial}{\partial t} \ln \right| X||=\frac{1}{2}\left|\frac{\partial}{\partial t} \ln g(X, X)\right| \leq M
$$

\footnotetext{
${ }^{1}$ Partly supported by an EPSRC advanced research fellowship.
} 
and so, for $t \in[0, T]$

$$
\operatorname{diam}(\mathcal{M}, g(0)) e^{-M t} \leq \operatorname{diam}(\mathcal{M}, g(t)) \leq \operatorname{diam}(\mathcal{M}, g(0)) e^{M t} .
$$

(See [3, Section 17] for improved estimates on how distances can shrink.) Unfortunately, if a Ricci flow develops a singularity in finite time, then the Ricci curvature must be unbounded (see [8]) so this diameter estimate degenerates as we take $T$ closer to the time of the singularity.

In this paper, we obtain improved control of the diameter of the flow, without assuming upper curvature bounds throughout. One should keep in mind that currently, it is still open as to whether the diameter must remain bounded over finite time intervals for the unnormalised flow (1.1), although severe constraints on any rate of blow-up can be established. The equivalent question for the normalised flow (1.2) is not as subtle; if one starts with the initial manifold $S^{n-1} \times S^{1}$, then at a later time $t$, one sees the manifold $S_{\varepsilon^{1 / n}}^{n-1} \times S_{\varepsilon^{-1+1 / n}}^{1}$, with $\varepsilon(t)=\left(1-\frac{2(n-2)}{n} t\right)^{\frac{n}{2}}$, where the subscripts denote the 'radii' of the spheres. The flow then clearly develops a singularity in finite time, and shortly before the singularity, the diameter is roughly $\pi \varepsilon^{-1+1 / n}$ which is blowing up. Nevertheless, it is possible to establish interesting estimates in this case also.

The starting point of the proof is Perelman's $\mathcal{W}$-entropy [6, Section 3] and we give an exposition of this theory using Perelman's notation, but with more detail added which could be compared to [4] and [10]. We use Perelman's ideas in conjuction with a new maximal function (see Section 4) which essentially allows us to develop a theory analagous to Perelman's 'no local collapsing' result [6, Section 4] replacing pointwise bounds for the full curvature tensor in Perelman's work by much weaker integral bounds on the scalar curvature, in any dimension.

\section{The estimates.}

We will give slightly different results for Ricci flow and normalised Ricci flow. However, it is not worth distinguishing the normalised flow if it differs only by a uniformly controlled homothetic scaling over finite time intervals, so we consider only normalised flows when the Ricci flow with the same initial metric has volume decreasing to zero in finite time.

Theorem 2.1. For $n \geq 3$, let $\left(\mathcal{M}^{n}, g(t)\right)$ be a smooth closed normalised Ricci flow (that is, a solution to (1.2)) on a maximal time interval $[0, T)$ with $0<T \leq \infty$, and suppose that the volume of the Ricci flow with the same 
initial metric converges to zero in finite time. Then there exists $C=C(g(0))$ such that

$$
\operatorname{diam}\left(\mathcal{M}^{n}, g(t)\right) \leq C \int_{\mathcal{M}}|R|^{\frac{n-1}{2}} d V,
$$

for $t$ sufficiently close to $T$ (or $t$ sufficiently large in the case that $T=\infty$ ) where $R$ and $d V$ are computed with respect to $g(t)$.

Remark 2.2. This estimate is sharp, as can be seen via the flow of $S^{n-1} \times$ $S^{1}$. We saw in the previous section that the flow at a later time is $S_{\varepsilon^{1 / n}}^{n-1} \times$ $S_{\varepsilon^{-1+1 / n}}^{1}$ with $\varepsilon(t) \downarrow 0$ as $t \uparrow T$. For small $\varepsilon$, the diameter is then roughly $\pi \varepsilon^{-1+1 / n}$ and since the scalar curvature is $C(n)\left(\varepsilon^{1 / n}\right)^{-2}$, the right-hand side of (2.1) may be computed to be $C(n) \varepsilon^{-1+1 / n}$.

Remark 2.3. Only the possibility of a flow which has $R \equiv 0$ at some instant in time forces us to require $t$ close to $T$. The dependence of $C$ on $g(0)$ implicitly includes dependence on $n$ and $\mathcal{M}$.

For the unnormalised flow, we require an extra hypothesis before claiming an estimate like (2.1) because of the possibility of starting with a flat manifold, which would not move under Ricci flow.

Theorem 2.4. For $n \geq 3$, let $\left(\mathcal{M}^{n}, g(t)\right)$ be a smooth closed Ricci flow (that is, a solution to (1.1)) on a time interval $[0, T)$ with $0<T<\infty$. Then there exists $C=C(g(0), T)$ such that for all $t \in[0, T)$, if $\operatorname{diam}\left(\mathcal{M}^{n}, g(t)\right) \geq C$, then

$$
\operatorname{diam}\left(\mathcal{M}^{n}, g(t)\right) \leq C \int_{\mathcal{M}}|R|^{\frac{n-1}{2}} d V
$$

with $R$ and $d V$ computed with respect to $g(t)$.

The proofs of these theorems will be given in Section 5 .

Remark 2.5. Theorem 2.4, as stated, only gives information in the case that the diameter blows up, since the constant $C$ is allowed to depend on $g(0)$. However, if one knew that the right-hand side of (2.2) remained finite under the Ricci flow, then one could deduce that the diameter remained bounded via a contradiction argument. Moreover, it is possible to determine the dependency of $C$ on $g(0)$ explicitly in terms of the dimension and elementary geometric quantities such as its volume, the infimum of its scalar curvature and its entropy (see Section 3). 
Remark 2.6. We will show in Section 5.3 that in the case that $n=3$, one can (after adjusting $C$ ) replace the conclusion (2.2) by the simpler conclusion

$$
\operatorname{diam}\left(\mathcal{M}^{n}, g(t)\right) \leq C \int_{\mathcal{M}} R d V
$$

This integral of $R$ - the total scalar curvature - turns out to be precisely the rate of decrease of volume, $-\frac{d V}{d t}$, under the Ricci flow (see for example [3] or [12]). In particular, if the diameter becomes unbounded under the flow, the estimate (2.3) forces volume to be dissipated at a controlled, unbounded rate.

Our results have some relation to diameter estimates for surfaces immersed in $\mathbb{R}^{3}$ as proved in [11, Lemma 1] and [9]. As we shall see, Perelman's entropy estimates give analogues of a key estimate from that work.

\section{Perelman's entropy.}

One of the ingredients of the proof is Perelman's $\mathcal{W}$-entropy [6]. Given a closed manifold $\mathcal{M}$ equipped with a Riemannian metric $g$, a function $f: \mathcal{M} \rightarrow \mathbb{R}$ and a number $\tau>0$, Perelman defines

$$
\mathcal{W}(g, f, \tau):=\int_{\mathcal{M}}\left[\tau\left(R+|\nabla f|^{2}\right)+f-n\right] u d V,
$$

where $u$ is defined by

$$
u:=(4 \pi \tau)^{-\frac{n}{2}} e^{-f} .
$$

We call the arguments $g, f$ and $\tau$ compatible if

$$
\int_{\mathcal{M}} u d V \equiv \int_{\mathcal{M}} \frac{e^{-f}}{(4 \pi \tau)^{\frac{n}{2}}} d V=1 .
$$

The following facts are required in the work of Perelman [6]. Prior work of Rothaus [7] provides detail for the proof of the first part.

Lemma 3.1. For any smooth Riemannian metric $g$ on $\mathcal{M}$, and $\tau>0$, the infimum of $\mathcal{W}(g, f, \tau)$ over all compatible $f$ (that is, those $f$ satisfying (3.2)) is attained by a smooth compatible $f$.

Defining $\mu$ as this infimum,

$$
\mu(g, \tau):=\inf _{f} \mathcal{W}(g, f, \tau),
$$

the function $\mu(g, \tau)$ is bounded below as $\tau$ varies within any finite interval $\left(0, \tau_{0}\right]$. 
Given this lemma, we may define, for $\tau_{0}>0$,

$$
\nu\left(g, \tau_{0}\right):=\inf _{\tau \in\left(0, \tau_{0}\right]} \mu(g, \tau) .
$$

Perelman has observed that $\mathcal{W}$ is monotonic under Ricci flow coupled with an appropriate flow for $f$ and $\tau$, provided a solution can be found:

Proposition 3.2. When $g, f$ and $\tau$ evolve according to

$$
\left\{\begin{array}{l}
\frac{\partial g}{\partial t}=-2 R i c \\
\frac{d \tau}{d t}=-1 \\
\frac{\partial f}{\partial t}=-\Delta f+|\nabla f|^{2}-R+\frac{n}{2 \tau}
\end{array}\right.
$$

the functional $\mathcal{W}$ increases, and

$$
\frac{d}{d t} \mathcal{W}(g, f, \tau)=2 \tau \int_{\mathcal{M}}\left|R i c+H e s s(f)-\frac{g}{2 \tau}\right|^{2} u d V \geq 0 .
$$

Remark 3.3. Under the evolution of the previous proposition, $u$ satisfies the linear backwards heat equation

$$
\square^{*} u:=-u_{t}-\Delta u+R u=0,
$$

One consequence is that the compatibility constraint (3.2) is preserved under the evolution in (3.5), because noting that $\frac{\partial}{\partial t} d V=-R d V$ (see, for example, [12]) we have

$$
\frac{d}{d t} \int_{\mathcal{M}} u d V=-\int_{\mathcal{M}} \square^{*} u d V=0
$$

We shall use the $\mathcal{W}$-entropy to prove the following result, which is a rephrasing of some of Perelman's work [6, Section 4]. With respect to a given metric, we use the notation $B(p, s)$ to denote the open geodesic ball centred at $p \in \mathcal{M}$ of radius $s>0$, and write

$$
V(p, s):=\operatorname{Vol}(B(p, s)) ; \quad K(p, s)=\frac{V(p, s)}{s^{n}},
$$

calling $K$ the volume ratio.

Theorem 3.4. Suppose $T_{0}>0, r_{0}>0$ and $p \in \mathcal{M}$. Then if $g(t)$ is a smooth Ricci flow for $t \in[0, T]$ with $T \in\left(0, T_{0}\right]$, and $r \in\left(0, r_{0}\right]$, then computing volumes, curvature and geodesic balls at time $T$, we have

$$
\gamma \leq \frac{V(p, r)}{V\left(p, \frac{r}{2}\right)}+\frac{r^{2}}{V\left(p, \frac{r}{2}\right)} \int_{B(p, r)}|R| d V+\ln [K(p, r)],
$$

for some $\gamma=\gamma\left(g(0), r_{0}, T_{0}\right) \in \mathbb{R}$. 
In the proof of this theorem, the monotonicity of $\mathcal{W}$ will give a lower bound for $\mathcal{W}$ under the flow, which will then be turned into geometric information via the following lemma.

Lemma 3.5. For any smooth metric $g$ on $\mathcal{M}, r>0, p \in \mathcal{M}$ and $\lambda>0$,

$$
\mu\left(g, \lambda r^{2}\right) \leq 36 \lambda \frac{V(p, r)}{V\left(p, \frac{r}{2}\right)}+\frac{\lambda r^{2}}{V\left(p, \frac{r}{2}\right)} \int_{B(p, r)}|R| d V+\ln \left[\frac{V(p, r)}{\left(4 \pi \lambda r^{2}\right)^{\frac{n}{2}}}\right]-n .
$$

The proof of such a result is hinted at in [6, Section 4]. Various expansions of those hints are available (see [4] or [10] for example). We give an alternative exposition in an appendix to this paper. One may set $\lambda=1$ in the lemma if one is prepared to accept a less clean version of (3.7).

Proof of Theorem 3.4. First, let us specialise Lemma 3.5 to the case $\lambda=\frac{1}{36}$ and $g=g(T)$, and estimate

$$
\mu\left(g(T), \frac{1}{36} r^{2}\right) \leq \frac{V(p, r)}{V\left(p, \frac{r}{2}\right)}+\frac{r^{2}}{V\left(p, \frac{r}{2}\right)} \int_{B(p, r)}|R| d V+\ln \left[\frac{V(p, r)}{r^{n}}\right]-\frac{n}{2} \ln \frac{\pi}{9}-n .
$$

By Lemma 3.1, there exists a smooth $f_{T}: \mathcal{M} \rightarrow \mathbb{R}$ compatible with $g(T)$ and $\tau=\frac{1}{36} r^{2}$ such that

$$
\mathcal{W}\left(g(T), f_{T}, \frac{1}{36} r^{2}\right)=\mu\left(g(T), \frac{1}{36} r^{2}\right) .
$$

We set $\tau=T+\frac{1}{36} r^{2}-t$, and for our given Ricci flow $g(t)$, find the $f: \mathcal{M} \times[0, T] \rightarrow \mathbb{R}$ with $f(T)=f_{T}$ completing a solution of (3.5). To see that this is possible, we may change variables from $f$ to $u$ which solves a backwards linear heat equation as discussed in Remark 3.3, where we also saw that $g(t), f(t)$ and $\tau$ remain compatible for all $t$. Using the definition of $\mu$, Proposition 3.2 and (3.9), we then have

$$
\begin{aligned}
& \mu\left(g(0), \frac{1}{36} r^{2}+T\right) \leq \mathcal{W}\left(g(0), f(0), \frac{1}{36} r^{2}+T\right) \leq \mathcal{W}\left(g(T), f(T), \frac{1}{36} r^{2}\right) \\
& \quad=\mu\left(g(T), \frac{1}{36} r^{2}\right),
\end{aligned}
$$

which coupled with (3.8) and the definition of $\nu$ from (3.4) gives

$$
\begin{aligned}
& \nu\left(g(0), \frac{1}{36} r_{0}^{2}+T_{0}\right)+\frac{n}{2} \ln \frac{\pi}{9}+n \\
& \quad \leq \frac{V(p, r)}{V\left(p, \frac{r}{2}\right)}+\frac{r^{2}}{V\left(p, \frac{r}{2}\right)} \int_{B(p, r)}|R| d V+\ln \left[\frac{V(p, r)}{r^{n}}\right] .
\end{aligned}
$$


Remark 3.6. One might compare Lemma 3.5 with [11, inequality (22)] and its predecessor in [9].

\section{The maximal function theorem.}

In Perelman's first approach to his 'no local collapsing' results, one uses a theorem such as Theorem 3.4 to control the volume ratio $K(p, r)$ from below, under pointwise constraints on the curvature tensor $|R m|$ (or more generally on the Ricci curvature). In contrast, we want to use Theorem 3.4 under weaker integral constraints on the scalar curvature. In this case, we can no longer control the collapsing directly at a given scale. However, we turn out to be able to use the same theorem to pass information on the amount of collapsing between different scales.

This technique will be used in the proof of Theorem 4.2 below, which is phrased in terms of the following new maximal function.

Definition 4.1. For smooth $f: \mathcal{M} \rightarrow \mathbb{R}$, we define the maximal function $M f: \mathcal{M} \times(0, \infty) \rightarrow \mathbb{R}$ of $f$ to be

$$
M f(p, r):=\sup _{s \in(0, r]} s^{-1}[V(p, s)]^{-\frac{n-3}{2}}\left(\int_{B(p, s)}|f| d V\right)^{\frac{n-1}{2}}
$$

Theorem 4.2. Suppose $n \geq 3, T_{0}>0, r_{0}>0$ and $\left(\mathcal{M}^{n}, g(t)\right)$ is a smooth closed Ricci flow (that is, a solution of (1.1)) for $t \in[0, T]$ with $T \in\left(0, T_{0}\right]$. Then there exists $\xi=\xi\left(g(0), r_{0}, T_{0}\right)>0$ such that for all $p \in \mathcal{M}$ and $r \in\left(0, r_{0}\right]$,

$$
K(p, r) \leq \xi \Longrightarrow M R(p, r)>\xi,
$$

when we compute quantities with respect to the metric $g(T)$.

This can be considered a refined version of the idea that we cannot simultaneously have small curvature and small volume ratio $K$.

We will prove this theorem with an explicit $\xi$. Let $\omega$ be the volume of the unit ball in $\mathbb{R}^{n}$. Given $r_{0}, T_{0}$ and an initial metric $g(0)$, let $\gamma$ be the number from Theorem 3.4. Then we set

$$
\xi:=\min \left\{\frac{\omega}{2}, e^{\gamma-2^{n+1}}\right\}
$$

Most of the proof of Theorem 4.2 will be contained in the following, describing how the property of small volume ratio $K$ is propagated to smaller scales when the curvature is small in a weak sense. 
Lemma 4.3. Given $n \geq 3, T_{0}>0, r_{0}>0$ and a smooth closed Ricci flow $\left(\mathcal{M}^{n}, g(t)\right)$ (that is, a solution of (1.1)) for $t \in[0, T]$ with $T \in\left(0, T_{0}\right]$, let us define $\xi$ as in (4.2). Computing with respect to the metric $g(T)$, if $M R(p, r) \leq \xi$ for some $p \in \mathcal{M}$ and $r \in\left(0, r_{0}\right]$, then for all $s \in(0, r]$,

$$
K(p, s) \leq \xi \Longrightarrow K\left(p, \frac{s}{2}\right) \leq \xi
$$

Before proving this lemma, we note how it implies the theorem.

Proof of Theorem 4.2. With $n, T_{0}, r_{0}, T$ and $g(t)$ satisfying the hypotheses of the theorem, suppose that the theorem is false with $\xi$ defined as in (4.2). Then there exist $p \in \mathcal{M}$ and $r \in\left(0, r_{0}\right]$ such that $K(p, r) \leq \xi$ and $M R(p, r) \leq \xi$. By applying Lemma 4.3, we learn that $K\left(p, \frac{r}{2}\right) \leq \xi$. Further applications tell us that $K\left(p, \frac{r}{4}\right) \leq \xi$, and indeed that for all $m \in \mathbb{N}$,

$$
K\left(p, 2^{-m} r\right) \leq \xi \leq \frac{\omega}{2}
$$

by (4.2). However, since $(\mathcal{M}, g(T))$ is a smooth Riemannian manifold, we must have $\lim _{s \downarrow 0} K(p, s)=\omega$, a contradiction.

Proof of Lemma 4.3. Suppose we are in the situation of the lemma, and that $M R(p, r) \leq \xi$ and $K(p, s) \leq \xi$ with $s \in(0, r]$. We will prove that $K\left(p, \frac{s}{2}\right) \leq \xi$ by considering two separate cases depending on the relative sizes of $V\left(p, \frac{s}{2}\right)$ and $V(p, s)$.

Case A. Suppose that $V\left(p, \frac{s}{2}\right) \leq \xi^{\frac{2}{n-1}} 2^{-n} s^{\frac{2 n}{n-1}}[V(p, s)]^{\frac{n-3}{n-1}}$.

Then we compute

$$
\begin{aligned}
K\left(p, \frac{s}{2}\right) & :=2^{n} s^{-n} V\left(p, \frac{s}{2}\right) \\
& \leq \xi^{\frac{2}{n-1}} s^{\frac{2 n}{n-1}-n}[V(p, s)]^{\frac{n-3}{n-1}} \\
& =\xi^{\frac{2}{n-1}}[K(p, s)]^{\frac{n-3}{n-1}} \\
& \leq \xi^{\frac{2}{n-1}} \xi^{\frac{n-3}{n-1}} \\
& =\xi
\end{aligned}
$$

as desired.

Case B. Suppose instead that $V\left(p, \frac{s}{2}\right)>\xi^{\frac{2}{n-1}} 2^{-n} s^{\frac{2 n}{n-1}}[V(p, s)]^{\frac{n-3}{n-1}}$. 
By definition of $M R(p, r)$, the fact that $M R(p, r) \leq \xi$ and the fact that we are in case $\mathrm{B}$, we have

$$
\begin{aligned}
\int_{B(p, s)}|R| d V & \leq[M R(p, r)]^{\frac{2}{n-1}} s^{\frac{2}{n-1}}[V(p, s)]^{\frac{n-3}{n-1}} \leq \xi^{\frac{2}{n-1}} s^{\frac{2}{n-1}}[V(p, s)]^{\frac{n-3}{n-1}} \\
& <2^{n} s^{-2} V\left(p, \frac{s}{2}\right),
\end{aligned}
$$

and combining with Perelman's work through Theorem 3.4, and the fact that $K(p, s) \leq \xi$, we then have

$$
\begin{aligned}
\gamma & \leq \frac{V(p, s)}{V\left(p, \frac{s}{2}\right)}+\frac{s^{2}}{V\left(p, \frac{s}{2}\right)} \int_{B(p, s)}|R| d V+\ln [K(p, s)] \\
& \leq \frac{V(p, s)}{V\left(p, \frac{s}{2}\right)}+2^{n}+\ln \xi
\end{aligned}
$$

By the definition of $\xi$ in (4.2), we know that $\ln \xi \leq \gamma-2^{n+1}$, giving

$$
2^{n} \leq \frac{V(p, s)}{V\left(p, \frac{s}{2}\right)}
$$

and hence,

$$
K\left(p, \frac{s}{2}\right)=\frac{2^{n} V\left(p, \frac{s}{2}\right)}{s^{n}} \leq \frac{V(p, s)}{s^{n}}=K(p, s) \leq \xi
$$

Remark 4.4. Weaker arguments along the lines of those in this section allow one to strengthen Perelman's no collapsing result [6, Section 4] to require only pointwise bounds on the scalar curvature rather than the full curvature tensor $|R m|$. See [12] for more details. Lei Ni has informed us that Perelman has also made this remark privately, and that related facts may be found in [5].

\section{Proofs of the results of Section 2 .}

\subsection{Proof of Theorem 2.1.}

Although this theorem concerns the normalised Ricci flow, we may exploit the scale-invariance of (2.1) and prove instead the following equivalent assertion. 
Claim 5.1. For $n \geq 3$, let $\left(\mathcal{M}^{n}, g(t)\right)$ be a smooth closed Ricci flow (that is, a solution to (1.1)) for $t \in\left[0, T_{0}\right)$ for which $\operatorname{Vol}(\mathcal{M}, g(t)) \rightarrow 0$ as $t \uparrow T_{0}<\infty$. Then there exists $C=C(g(0))$ such that

$$
\operatorname{diam}\left(\mathcal{M}^{n}, g(t)\right) \leq C \int_{\mathcal{M}}|R|^{\frac{n-1}{2}} d V
$$

for $t$ sufficiently close to $T_{0}$.

Proof of Claim 5.1. With the flow $g(t)$ and $T_{0}$ of the claim, and $r_{0}=1$, let $\xi$ be the number generated by Theorem 4.2. Since the volume converges to zero, for $T<T_{0}$ sufficiently close to $T_{0}$, we may assume that $\operatorname{Vol}(\mathcal{M}, g(T)) \leq$ $\xi$, and in particular that for all $p \in \mathcal{M}, K(p, 1)=V(p, 1) \leq \xi$. By Theorem 4.2 , we then find that $M R(p, 1)>\xi$ for all $p \in \mathcal{M}$. Unravelling the definition of the maximal function, we can then be sure that for all $p \in \mathcal{M}$, there exists $s=s(p)>0$ such that

$$
\xi \leq s^{-1}[V(p, s)]^{-\frac{n-3}{2}}\left(\int_{B(p, s)}|R| d V\right)^{\frac{n-1}{2}},
$$

and by Hölder's inequality,

$$
\xi \leq \frac{1}{s} \int_{B(p, s)}|R|^{\frac{n-1}{2}} d V
$$

We now have to pick appropriate points $p$ at which to apply (5.2). Let $a, b$ be extremal points in $\mathcal{M}$ at time $T$, in that the distance from $a$ to $b$ with respect to $g(T)$ achieves its maximum $\operatorname{diam}(\mathcal{M}, g(T))$. Let $\Sigma$ be a shortest geodesic between $a$ and $b$. Clearly, $\Sigma$ is covered by the balls $\{B(p, s(p)): p \in \Sigma\}$. However, we also have the following covering lemma.

Lemma 5.2. There exists a countable (possibly finite) set of points $\left\{p_{i}\right\} \subset \Sigma$ such that the balls $B\left(p_{i}, s\left(p_{i}\right)\right)$ are disjoint, and cover at least one third of $\Sigma$.

Assuming this lemma for the moment, we have

$$
\frac{1}{3} \operatorname{diam} \leq \text { length }\left(\bigcup_{i} \Sigma \cap B\left(p_{i}, s\left(p_{i}\right)\right)\right) \leq \sum_{i} 2 s\left(p_{i}\right),
$$


and by (5.2), we may then conclude the proof of Claim 5.1 with

$$
\begin{aligned}
\operatorname{diam}(\mathcal{M}, g(T)) & \leq 6 \sum_{i} s\left(p_{i}\right) \leq 6 \sum_{i} \frac{1}{\xi} \int_{B\left(p_{i}, s\left(p_{i}\right)\right)}|R|^{\frac{n-1}{2}} d V \\
& \leq \frac{6}{\xi} \int_{\mathcal{M}}|R|^{\frac{n-1}{2}} d V .
\end{aligned}
$$

For completeness, we give a proof of the covering lemma.

Proof of Lemma 5.2. First pick $p_{1} \in \Sigma$ so that $s\left(p_{1}\right) \geq \frac{1}{2} \sup _{p \in \Sigma} s(p)$. We define the rest of the sequence $\left\{p_{i}\right\}$ inductively. For $i \in \mathbb{N}$, define

$$
\Omega_{i}:=\left\{p \in \Sigma: B(p, s(p)) \text { is disjoint from } B\left(p_{j}, s\left(p_{j}\right)\right) \text { for } 1 \leq j \leq i\right\} .
$$

Given $p_{1}, \ldots, p_{i}$, we pick $p_{i+1} \in \Omega_{i}$ such that

$$
s\left(p_{i+1}\right) \geq \frac{1}{2} \sup _{p \in \Omega_{i}} s(p) .
$$

This inductive point-picking process may go on forever (in which case $s\left(p_{i}\right) \rightarrow 0$ ) or may terminate if $\Omega_{i}$ becomes empty. Either way, we can be sure that for any $q \in \Sigma$, there must be non-trivial intersection between $B(q, s(q))$ and at least one of the balls $B\left(p_{i}, s\left(p_{i}\right)\right)$. Indeed, if this were not the case, then surely the point picking process did not terminate (because we could pick $q$ as the next point in the sequence), but since $s\left(p_{i}\right) \rightarrow 0$, and we could at any time have picked $q$ (that is, $q \in \Omega_{i}$ for all $i$ ) we cannot have performed the point-picking to correctly satisfy (5.3).

Now, we assert that

$$
\Sigma \subset \bigcup_{i} B\left(p_{i}, 3 s\left(p_{i}\right)\right)
$$

Indeed, for an arbitrary point $q \in \Sigma$, we saw above that $B(q, s(q))$ must intersect at least one of the balls $B\left(p_{i}, s\left(p_{i}\right)\right)$, so let us fix the least such $i$ for which this is true. (Note that if $i>1$, then $q \in \Omega_{i-1}$.) By virtue of the point picking rule (5.3), we must have $s\left(p_{i}\right) \geq \frac{1}{2} s(q)$, and hence since $B(q, s(q))$ and $B\left(p_{i}, s\left(p_{i}\right)\right)$ intersect,

$$
\operatorname{dist}\left(q, p_{i}\right) \leq s(q)+s\left(p_{i}\right) \leq 3 s\left(p_{i}\right)
$$

which shows that $q \in B\left(p_{i}, 3 s\left(p_{i}\right)\right)$, establishing (5.4). 
Consequently, we may conclude the proof of the covering lemma with the calculation

$$
\begin{aligned}
\text { length }(\Sigma) & \leq \sum_{i} \text { length }\left(\Sigma \cap B\left(p_{i}, 3 s\left(p_{i}\right)\right)\right) \\
& \leq 3 \sum_{i} \text { length }\left(\Sigma \cap B\left(p_{i}, s\left(p_{i}\right)\right)\right) \\
& =3 \text { length }\left(\Sigma \cap\left(\bigcup_{i} B\left(p_{i}, s\left(p_{i}\right)\right)\right)\right)
\end{aligned}
$$

\subsection{Proof of Theorem 2.4.}

In order to match the notation of the theorem with the notation of the tools we have developed, we record the following equivalent formulation of Theorem 2.4.

Claim 5.3. For $n \geq 3$, let $\left(\mathcal{M}^{n}, g(t)\right)$ be a smooth closed Ricci flow (that is, a solution to (1.1)) on a time interval $[0, T]$ with $0<T \leq T_{0}<\infty$. Then there exists $C=C\left(g(0), T_{0}\right)$ such that if $\operatorname{diam}(\mathcal{M}, g(T)) \geq C$, then with respect to $g(T)$,

$$
\operatorname{diam}(\mathcal{M}, g(T)) \leq C \int_{\mathcal{M}}|R|^{\frac{n-1}{2}} d V .
$$

In contrast to Theorem 2.4, we no longer ask that the volume decays to zero, and thus cannot assume that $K(p, 1)=V(p, 1) \leq \xi$ for all $p$. However, we can control the set of relevant points for which this inequality fails. Indeed, we have the following lemma which allows us to complete the proof using precisely the same method as in the proof of Theorem 2.1, but with the modified $\Sigma$ as below.

Lemma 5.4. For $n \geq 3$, let $\left(\mathcal{M}^{n}, g(t)\right)$ be a smooth closed Ricci flow (that is, a solution to (1.1)) on a time interval $[0, T]$ with $0<T \leq T_{0}<\infty$. Set $r_{0}=1$, and let $\xi$ be defined by (4.2) (which then depends on $g(0)$ and $T_{0}$ ).

There exist constants $D$ and $\beta$ depending on $g(0)$ and $T_{0}$ such that if $\operatorname{diam}(\mathcal{M}, g(T)) \geq D$, then with respect to $g(T)$, there exists a minimising geodesic $\Sigma$ connecting two points in $\mathcal{M}$ with the properties that

- for all $p \in \Sigma, K(p, 1) \leq \xi$; 
- $\operatorname{diam}(\mathcal{M}, g(T)) \leq \beta$ length $(\Sigma)$.

Proof. Let $R_{0}$ be the minimum of the scalar curvature $R$ at time $t=0$. It is well known and easy to prove (see [3] or [12] for example) that at all points in $\mathcal{M}$ and at all subsequent times $t \in[0, T]$, we have $R \geq R_{0}$. Meanwhile, as we mentioned in Remark 2.6, if $V(t):=\operatorname{Vol}(\mathcal{M}, g(t))$ then

$$
\frac{d V}{d t}=-\int_{\mathcal{M}} R d V \leq-R_{0} V
$$

and, in particular, we have

$$
V(T) \leq \bar{V}:=e^{\left|R_{0}\right| T_{0}} V(0),
$$

an upper bound depending only on $g(0)$ and $T_{0}$.

From now on, we work exclusively with respect to the metric $g(T)$. Choose $a, b \in \mathcal{M}$ such that $\operatorname{dist}(a, b)=\operatorname{diam} \mathcal{M}$. Let $\sigma$ be a minimising geodesic from $a$ to $b$. If $K(p, 1) \leq \xi$ for all $p \in \sigma$, then we set $\Sigma=\sigma$, and the lemma holds for any $D$, provided $\beta \geq 1$. Otherwise, we will construct the $\Sigma$ of the lemma to be a (connected) subset of $\sigma$. Pick points $p_{1}, \ldots, p_{N} \in \sigma$ such that the balls $\left\{B\left(p_{i}, 1\right)\right\}$ are disjoint and $V\left(p_{i}, 1\right)=K\left(p_{i}, 1\right)>\xi$, with $N$ as large as possible. Clearly $N \xi \leq \bar{V}$.

We define $\Sigma$ to be a largest component of the set $\sigma \backslash \bigcup_{i=1}^{N} B\left(p_{i}, 2\right)$, which may have up to $N+1$ disconnected components. (By asking at least that $D>\frac{4 \bar{V}}{\xi}$, we can be sure that this set is not empty because then by hypothesis, length $(\sigma) \geq D>\frac{4 \bar{V}}{\xi} \geq 4 N$.) Then for all $p \in \Sigma$, we must have $K(p, 1) \leq \xi$ since otherwise, we contradict the maximality of $N$. Moreover,

$$
\begin{aligned}
\operatorname{diam}(\mathcal{M}, g(T)) & \leq(N+1) \text { length }(\Sigma)+4 N \\
& \leq\left(\frac{\bar{V}}{\xi}+1\right) \text { length }(\Sigma)+4 \frac{\bar{V}}{\xi} \\
& \leq\left(\frac{\bar{V}}{\xi}+1\right) \text { length }(\Sigma)+\frac{D}{2},
\end{aligned}
$$

if we ask that $D \geq \frac{8 \bar{V}}{\xi}$. Therefore, if diam $\geq D$, we have

$$
\operatorname{diam}(\mathcal{M}, g(T)) \leq 2\left(\frac{\bar{V}}{\xi}+1\right) \text { length }(\Sigma),
$$

completing the proof provided that $\beta \geq 2\left(\frac{\bar{V}}{\xi}+1\right)$. 


\subsection{Justification of Remark 2.6.}

In the case $n=3$, the conclusion of Theorem 2.4 is an estimate

$$
\operatorname{diam} \leq C \int_{\mathcal{M}}|R| d V
$$

We wish to show that one can replace $|R|$ in this estimate by $R$, at the expense of a larger constant $C$. As in the previous section, we will use the fact that the volume has a bound $V(t) \leq \bar{V}$ depending on $g(0)$ and $T$ (with $T$ being an upper bound for $t$ as in Theorem 2.4) and that the scalar curvature $R$ satisfies $R \geq R_{0}$ at all points and times, where $R_{0}$ is the minimum scalar curvature initially. Then $|R| \leq R+2\left|R_{0}\right|$, and so

$$
\int_{\mathcal{M}}|R| d V \leq \int_{\mathcal{M}} R d V+2\left|R_{0}\right| \bar{V}
$$

By the hypothesis diam $\geq C$ of Theorem 2.4 and its conclusion (2.2), after increasing $C$, we can ask for the additional conclusion that

$$
4\left|R_{0}\right| \bar{V} \leq \int_{\mathcal{M}}|R| d V
$$

and hence that

$$
\int_{\mathcal{M}}|R| d V \leq 2 \int_{\mathcal{M}} R d V
$$

\section{Appendix: the proof of Lemma 3.5.}

Our approach below was influenced by conversations with Klaus Ecker.

Proof of Lemma 3.5 - c.f. [6, Section 4]. If we adopt the change of variables $\phi=e^{-\frac{f}{2}}$, and write $\tau=\lambda r^{2}$, then we find (abusing notation for $\mathcal{W}$ ) that

$$
\mathcal{W}\left(g, \phi, \lambda r^{2}\right)=\left(4 \pi \lambda r^{2}\right)^{-\frac{n}{2}} \int_{\mathcal{M}}\left[\lambda r^{2}\left(4|\nabla \phi|^{2}+R \phi^{2}\right)-2 \phi^{2} \ln \phi-n \phi^{2}\right] d V
$$

and the compatibility constraint (3.2) becomes

$$
\left(4 \pi \lambda r^{2}\right)^{-\frac{n}{2}} \int_{\mathcal{M}} \phi^{2} d V=1
$$

These expressions have the benefit of making perfect sense when $\phi$ is merely weakly (rather than strictly) positive, and by approximation,

$$
\inf _{f} \mathcal{W}\left(g, f, \lambda r^{2}\right)=\inf _{\phi} \mathcal{W}\left(g, \phi, \lambda r^{2}\right),
$$


where the infima are taken over $f: \mathcal{M} \rightarrow \mathbb{R}$ and $\phi: \mathcal{M} \rightarrow[0, \infty)$ compatible with $g$ and $\tau=\lambda r^{2}$, and we are abusing notation for $\mathcal{W}$ as usual.

Let $\psi:[0, \infty) \rightarrow[0,1]$ be a smooth cut-off function, supported in $[0,1]$, such that $\psi(y)=1$ for $y \in\left[0, \frac{1}{2}\right]$ and $\left|\psi^{\prime}\right| \leq 3$. We then write

$$
\phi(x)=e^{-\frac{c}{2}} \psi\left(\frac{\operatorname{dist}(x, p)}{r}\right),
$$

where $c \in \mathbb{R}$ is determined by the constraint (6.2), and since

$$
V\left(p, \frac{r}{2}\right) \leq e^{c} \int_{\mathcal{M}} \phi^{2} d V \leq V(p, r)
$$

we deduce that

$$
\left(4 \pi \lambda r^{2}\right)^{-\frac{n}{2}} V\left(p, \frac{r}{2}\right) \leq e^{c} \leq\left(4 \pi \lambda r^{2}\right)^{-\frac{n}{2}} V(p, r) .
$$

We now estimate each of the four terms in (6.1) separately.

Term 1. For the specific $\phi$ we have chosen, whose gradient is supported on $B(p, r) \backslash B\left(p, \frac{r}{2}\right)$, and satisfies $|\nabla \phi| \leq e^{-\frac{c}{2}} \frac{1}{r} \sup \left|\psi^{\prime}\right| \leq \frac{3}{r} e^{-\frac{c}{2}}$, we estimate

$$
\begin{aligned}
\left(4 \pi \lambda r^{2}\right)^{-\frac{n}{2}} \int_{\mathcal{M}} \lambda r^{2} 4|\nabla \phi|^{2} d V & \leq 4 \lambda r^{2}\left(4 \pi \lambda r^{2}\right)^{-\frac{n}{2}} \sup |\nabla \phi|^{2} V(p, r) \\
& \leq 36 \lambda\left(4 \pi \lambda r^{2}\right)^{-\frac{n}{2}} e^{-c} V(p, r) \\
& \leq 36 \lambda \frac{V(p, r)}{V\left(p, \frac{r}{2}\right)}
\end{aligned}
$$

the last inequality using the first part of (6.4).

Term 2. For the specific $\phi$ we have chosen, which is supported on $B(p, r)$, and satisfies

$$
\phi^{2} \leq e^{-c} \leq \frac{\left(4 \pi \lambda r^{2}\right)^{\frac{n}{2}}}{V\left(p, \frac{r}{2}\right)}
$$

we estimate,

$$
\left(4 \pi \lambda r^{2}\right)^{-\frac{n}{2}} \int_{\mathcal{M}} \lambda r^{2} R \phi^{2} d V \leq \frac{\lambda r^{2}}{V\left(p, \frac{r}{2}\right)} \int_{B(p, r)}|R| d V .
$$

Term 3. By again using the fact that the support of $\phi$ lies within $B(p, r)$, we rewrite

$$
\left(4 \pi \lambda r^{2}\right)^{-\frac{n}{2}} \int_{\mathcal{M}}-2 \phi^{2} \ln \phi d V=\int G(\sigma) d \mu
$$


where $\sigma: B(p, r) \rightarrow[0, \infty)$ is defined by $\sigma:=\phi^{2}$, the continuous function $G:[0, \infty) \rightarrow \mathbb{R}$ is defined for $y>0$ by $G(y):=-y \ln y$, and the measure $d \mu$ is supported on $B(p, r)$, where $d \mu:=\left(4 \pi \lambda r^{2}\right)^{-\frac{n}{2}} d V$. Because $G$ is concave, we may apply Jensen's inequality:

$$
f G(\sigma) d \mu \leq G(f \sigma d \mu)
$$

(see for example [13, Theorem 1.7]) and since by (6.2), we have

$$
\int \sigma d \mu=1
$$

this tells us that

$$
\int G(\sigma) d \mu \leq\left(\int d \mu\right) G\left(\frac{1}{\int d \mu}\right)=\ln \left(\int d \mu\right) .
$$

By (6.5) and the definition of $d \mu$, we conclude that

$$
\left(4 \pi \lambda r^{2}\right)^{-\frac{n}{2}} \int_{\mathcal{M}}-2 \phi^{2} \ln \phi d V \leq \ln \left[\frac{V(p, r)}{\left(4 \pi \lambda r^{2}\right)^{\frac{n}{2}}}\right] .
$$

Term 4. By the constraint (6.2), we have simply

$$
\left(4 \pi \lambda r^{2}\right)^{-\frac{n}{2}} \int_{\mathcal{M}}-n \phi^{2} d V=-n
$$

When we combine these calculations with (6.1), we find that for the particular $\phi$ under consideration,

$$
\mathcal{W}\left(g, \phi, \lambda r^{2}\right) \leq 36 \lambda \frac{V(p, r)}{V\left(p, \frac{r}{2}\right)}+\frac{\lambda r^{2}}{V\left(p, \frac{r}{2}\right)} \int_{B(p, r)}|R| d V+\ln \left[\frac{V(p, r)}{\left(4 \pi \lambda r^{2}\right)^{\frac{n}{2}}}\right]-n,
$$

which together with (6.3), proves the lemma.

\section{References.}

[1] D. M. DETURCK, Deforming metrics in the direction of their Ricci tensors (improved version). Collected papers on the Ricci flow. eds H.D.Cao, B.Chow, S.C.Chu, S.T.Yau. Series in geometry and topology 37 International Press (2003) 163-165.

[2] R. S. HAMILTON, Three manifolds with positive Ricci curvature. J. Diff. Geom. 17 (1982) 255-306. 
[3] R. S. HAMILTON, The formation of singularities in the Ricci flow. Surveys in Diff. Geom. 2 (1995) 7-136.

[4] B. KLEINER and J. LOTT, Notes on Perelman's papers. (2003) http://www.math.lsa.umich.edu/research/ricciflow/perelman.html

[5] L. NI, The entropy formula for linear heat equation. J. Geom. Anal. 14 (2004) 87-100; Addendum. J. Geom. Anal. 14 (2004) 369-374.

[6] G. PERELMAN, The entropy formula for the Ricci flow and its geometric applications. http://arXiv.org/abs/math.DG/0211159

[7] O. S. ROTHAUS, Logarithmic Sobolev inequalities and the spectrum of Schrödinger operators. J. Funct. Anal. 42 (1981) 110-120.

[8] N. SESUM, Curvature tensor under the Ricci flow. http://arXiv.org/abs/math.DG/0311397

[9] L. M. SIMON, Existence of surfaces minimizing the Willmore functional. Comm. Anal. Geom. 1 No.2 (1993) 281-326.

[10] N. SESUM, G. TIAN and X. WANG Notes on Perelman's paper on the entropy formula for the Ricci flow and its geometric applications. (2003)

[11] P. M. TOPPING, Mean Curvature Flow and Geometric Inequalities. J. Reine Angew. Math. 503 (1998) 47-61.

[12] P. M. TOPPING, Lectures on the Ricci flow. Course notes from spring 2004, to appear, LMS Lecture Notes, C.U.P. (2006).

[13] S. R. S. VARADHAN, Probability Theory. Courant lecture notes in mathematics 7 AMS (2001).

Mathematics Institute

UNIVERSITY OF WARWICK

Coventry, CV4 7AL

U.K.

http://www. maths. warwick.ac.uk/ topping/

Received August 25, 2004. 